\title{
DOENÇAS SEXUALMENTE TRANSMISSÍVEIS \\ Uso de Imiquimode Tópico no Tratamento da Infecção Anal pelo Papilomavírus Humano
}

\author{
The Use of Topic Imiquimod in the Treatment of the Anal Infection \\ by Human Papillomavirus
}

CARMEN RUTH MANZIONE, TSBCP; FERNANDA BELLOTTI FORMIGA, ASBCP; SIDNEY ROBERTO NADAL, TSBCP

MANZIONE CR; FORMIGA FB; NADAL SR. Uso de Imiquimode Tópico no Tratamento da Infecção Anal pelo Papilomavírus Humano. Rev bras Coloproct, 2010;30(1): 092-094.

RESUMO: Dos diversos tratamentos da infecção anal pelo papilomavírus humano, uma opção é o imunomodulador imiquimode. Derivado da família imidazoquinolina, o imiquimode é quimioterápico e imuno-estimulante com atividade antitumoral e antiviral. A medicação é aplicada em esquema domiciliar, três vezes por semana em noites alternadas, por oito a 16 semanas. Os efeitos adversos locais são comuns, mas bem tolerados. A droga atinge remissão de 74 a $84 \%$, sendo completa entre 25 e $77 \%$ dos doentes, com menor taxa de remissão completa e maior índice de recidiva em imunodeprimidos. Aguardamos estudos com grandes casuísticas para avaliar melhor a eficácia dessa medicação, incluindo a incidência de recidivas e o tempo livre de novas lesões.

Descritores: Infecções por Papillomavirus; Imunoterapia, efeitos adversos; Condiloma acuminado, terapia; Imunomodulação, efeito de drogas.

Há diversos tratamentos para a infecção anal pelo papilomavírus humano (HPV). Os mais comuns são as medicações tópicas (podofilina, ácido tricloroacético e podofilotoxina), os procedimentos cirúrgicos (ablação elétrica, LASER, coagulação pelo infravermelho, crioterapia e excisão cirúrgica) e os imunomoduladores (imiquimode, resiquimode e interferon).

O imiquimode, derivado da família imidazoquinolina (1-[2-metilpropil)-1H-imidazo-[4,5-c]quinolin-4-amina), é quimioterápico e imuno-estimulante com atividade antitumoral e antiviral. ${ }^{(1,2)}$ Tem ação imunomoduladora, através da atividade agonista no receptor 7 dos monócitos, macrófagos e células dendríticas (Langerhans), ativando a imunidade inata e a celular (Th1) pela indução das citocinas pró-inflamatórias como interferon alfa, fator de necrose tumoral alfa e interleucinas 1, 6, 8 e 12. Além disso, induz apoptose e ativa os linfócitos B, potencializando a resposta imunológica contra as células alteradas pelo HPV. ${ }^{(3-11)}$
Aprovado para tratamento de condiloma anogenital em 1997, ${ }^{(12)}$ foi liberado no Brasil pela Agência Nacional de Vigilância Sanitária (ANVISA) para uso em maiores de 12 anos e recomendado para aplicação externa. Porém, há descrição na literatura de uso em criança de nove anos com regressão completa da lesão condilomatosa. ${ }^{(13)} \mathrm{O}$ imiquimode na gestação é considerado categoria B pelo Food and Drug Administration, não sendo recomendado por não haver estudos controlados em gestantes. O resiquimode, derivado do mesmo grupo farmacológico, ainda não teve seu uso autorizado.

A medicação é aplicada (após instrução médica) em esquema domiciliar, digitalmente em toda borda anal, pela própria pessoa ou acompanhante. Aplica-se um envelope três vezes por semana, em noites alternadas, antes de dormir, lavando-se bem pela manhã, durante oito a 16 semanas.

Algum grau de eritema local ocorre naqueles que aderem ao tratamento, decorrente da liberação das citocinas pró-inflamatórias, como parte do mecanismo

Trabalho realizado pela Equipe Técnica de Proctologia do Instituto de Infectologia Emílio Ribas - São Paulo - Brasil.

Recebido em 22/10/2009

Aceito para publicação 02/12/2009 
de ação da droga. ${ }^{(1)}$ Efeitos adversos ocorrem em 50\% dos usuários da medicação, sendo a maioria deles efeitos locais como vermelhidão, queimação, irritação, ulceração e dor. Os efeitos sistêmicos (3 a 18\%) mais comuns são a síndrome gripal, a cefaléia, as alterações do trato gastro-intestinal e as tonturas. ${ }^{(1,2)}$

O imiquimode promove redução qualitativa (diminuição de cepas mais virulentas) e quantitativa (redução do número dos tipos infectantes que co-existem) do HPV, diferente das outras formas de tratamento que não são imunomoduladoras. . $^{(1,2,14,15)}$

Embora a eficácia da medicação e sua melhor indicação venham sendo muito estudadas, não há padronização na literatura nem estudos prospectivos controlados, e com amostras homogêneas, com grau de evidência necessário para justificar seu uso como primeira linha no tratamento das lesões clínicas induzidas pelo HPV. Aliado a isso, o custo ainda é elevado em relação a outros esquemas tópicos.

Alguns estudos comparando técnicas ablativas (cirurgia e infravermelho) com o imiquimode, como primeira opção de tratamento, mostraram maior tempo livre de doença com o imunomodulador. ${ }^{(2,16-21)}$ Já, comparando o tratamento tópico convencional (podofilina) com o imiquimode, observou-se a mesma efetividade em relação ao índice de cura, porém, com maior custo do segundo. ${ }^{(22)}$

Os condilomas acuminados anogenitais tratados com imiquimode mostraram remissão entre 74 e $84 \%$, sendo completa entre 25 e $77 \%$ dos doentes. ${ }^{(1,2,20,23,24)}$ Em imunocompetentes, atingiu 50 a $64 \%$ de resposta completa com até 16 semanas de tratamento e $13 \%$ de recidivas. ${ }^{(24-26)}$ Já, os imunodeprimidos mostraram menores taxas de remissão completa (31 a $46 \%$ ), com índice de recidiva maior $(29 \%){ }^{(20,27)}$

Considerando tudo isso, em nosso serviço, optamos pelo uso do imiquimode em pacientes com lesões multirrecidivadas ${ }^{(3,28)}$ e, como primeira opção, na papulose bowenóide. ${ }^{(29)} \mathrm{Na}$ prática, são os doentes que já receberam o tratamento tópico (podofilina na margem anal e ácido tricloroacético no canal anal) por quatro a oito semanas, sem sucesso, que foram submetidos a exérese cirúrgica, que recidivaram e receberam novo tratamento tópico ou sofreram nova operação. Na nova recidiva, indicamos o imunomodulador, na tentativa de melhorar os fatores inflamatórios locais e conferir imunidade aos tipos virais envolvidos. De qualquer forma, precisamos de estudos com grandes casuísticas para avaliar melhor a eficácia dessa medicação, incluindo a incidência de recidivas e o tempo livre de novas lesões.

ABSTRACT: Considering several kinds of treatments for human papillomavirus anal infection, the topical immune response modifier imiquimod is an option. An imidazoquinoline derivate, imiquimod is a chemotherapic drug and an immune stimulator with antitumoral and antiviral action. The medication is used at home, by patients, three times a week, in alternated nights, for eight to 16 weeks. Side-effects are common, but well tolerated. This drug reaches from 74 to $84 \%$ of remission, although from 25 to $77 \%$ are complete. There are lower complete remission and higher recurrence in immune compromised patients. Studies with more patients for better evaluation of this medication efficiency are needed, including those about recurrences and time free from new lesions.

Key words: Papillomavirus infections; Immunotherapy, adverse effects; Condylomata Acuminata, therapy; Immunomodulation, drug effects.

\section{REFERÊNCIAS}

1. Wieland U, Brockmeyer NH, Weissenborn SJ, Hochdorfer B, Stücker M, Swoboda J et al. Imiquimod treatment of anal intraepithelial neoplasia in HIV-positive men. Arch Dermatol 2006;142:1438-44.

2. Kreuter A, Potthoff A, Brockmeyer NH, Gambichler T, Stücker M, Altemeyer Pet al. Imiquimod leads to a decrease of human papillomavirus DNA and to a sustained clearance of anal intraepithelial neoplasia in HIV-infected men. J Invest Dermatol 2008; 128:2078-83.
3. Nadal SR, Manzione CR, Horta SHC, Calore EE. Sistematização do atendimento dos portadores de infecção perianal pelo Papilomavirus humano (HPV). Rev bras Coloproct 2004; 24(4):322-328.

4. Gupta AK, Browne M, Bluhm R. Imiquimod: a review. J Cutan Med Surg 2002; 6(6):554-60.

5. Pehoushek J, Smith KJ. Imiquimod and 5\% Fluorouracil therapy for anal and perianal squamous cell carcinoma in situ in an HIV-1 positive man. Arch Dermatol 2001; 137:14-16.

6. Hengge UR, Borchard C, Esser S, Schroder M, Mirmohammadsadegh A, Goss M. Lymphocytes proliferative 
in blood and lymph nodes following interleukin-2 therapy in addition to highly active antiretroviral therapy. AIDS 2002; 16:151-60.

7. Hengge UR, Ruzicka T. Topical immunomodulation on in dermatology: potential of toll-like receptor agonists. Dermatol Surg 2004; 30:1101-1112.

8. Garland SM. Imiquimod. Curr Opin Infect Dis 2003; 16:8589.

9. Hengge UR, Benninghoff B, Ruzicka T, Gooss M. Topical immunomodulators - progress towards treating inflammatio, infection, and cancer. Lancet Infect Dis 2001; 1:189-198.

10. Mcinturff JE, Modlin RL, Kim J. The role of toll-like receptors in the pathogenesis and treatment of dermatological disease. $\mathrm{J}$ Invest Dermatol 2005; 125:1-8.

11. Meyer T, Nindl I, Schmook T, Ulrich C, Sterry W, Stockfleth E. Induction of apoptosis by Toll-like receptor-7 agonist in tissue cultures. Br J Dermatol 2003; 149 (suppl 66):9-14.

12. Schöfer $\mathrm{H}$. Evaluation of imiquimod for the therapy of external genital and anal warts in comparison with destructive therapies. Br J Dermatol 2007;157 Suppl 2:52-5.

13. Godfrey JC, Vaughan MC, Williams JV. Successful treatment of bowenoid papulosis in a 9-year-old girl with vertically acquired human immunodeficiency virus. Pediatrics 2003;112:73-6.

14. Kreuter A, Wieland U, Gambichler T, Altmeyer P, Pfister H, Tenner-Racz K et al. p16ink4a expression decreases during imiquimod treatment of anal intraepithelial neoplasia in human immunodeficiency virus-infected men and correlates with the decline of lesional high-risk human papillomavirus DNA load. Br J Dermatol 2007; 157(3):523-30.

15. Kreuter A, Brockmeyer NH, Weissenborn SJ, Wafaisade A, Pfister H, Altmeyer P et al. 5\% imiquimod suppositories decrease the DNA load of intra-anal HPV types 6 and 11 in HIV-infected men after surgical ablation of condylomata acuminata. Arch Dermatol 2006; 142(2):243-4.

16. Chang GJ, Berry JM, Jay N, Palefsky JM, Welton ML. Surgical treatment of high-grade anal squamous intraepithelial lesions: a prospective study. Dis Colon Rectum 2002; 45:453458.

17. Pineda CE, Berry JM, Jay N, Palefsky JM, Welton ML. High-resolution anoscopy targeted surgical destruction of anal high-grade squamous intraepithelial lesions: a ten-year experience. Dis Colon Rectum 2008; 51:829-35.

18. Goldstone SE, Kawalek AZ, Huyett JW. Infrared coagulation: a useful toll of high-grade anal squamous intraepithelial lesions. Dis Colon Rectum 2005; 48:1042-1054.

19. Cranston RD, Hirschowitz SL, Cortina G, Moe AA. A retrospective clinical study of the treatment of high-grade anal dysplasia by infrared coagulation in a population of HIVpositive men who have sex with men. Int J STD AIDS 2008; 19:118-20.

20. Sanclemente G, Herrera S, Tyring SK, Rady PL, Zuleta JJ, Correa LA et al. Human papillomavirus (HPV) viral load and HPV type in the clinical outcome of HIV-positive patients treated with imiquimod for anogenital warts and anal intraepithelial neoplasia. J Eur Acad Dermatol Venereol 2007; 21:1054-60.

21. Schöfer H, Van Ophoven A, Henke U, Lenz T, Eul A. Randomized, comparative trial on the sustained efficacy of topical imiquimod 5\% cream versus convencional ablative methods in external anogenital warts. Eur J Dermatol 2006;16(6):642-8.

22. Williams P, von Krogh G. The cost-effectiveness of patientapplied treatments for anogenital warts. Int J STD AIDS 2003; 14(4):228-34.

23. Nadal SR, Nadal LRM, Horta SR, Manzione CR. Efeitos da contagem sérica dos linfócitos T CD4+ na eficácia do imiquimod nos condilomas anais recidivantes de doentes HIV-positivo. Rev bras Coloproct 2006; 26(supl.):41.

24. Senatori R, Dionisi B, Lippa P, Inghirami P. Topical imiquimod cream in the treatment of external anogenital warts: personal experience. Minerva Ginecol 2003; 55(6):541-6.

25. Arican O, Guneri F, Bilgic K, Karaoglu A. Topical imiquimod $5 \%$ cream in external anogenital warts: a randomized, doubleblind, placebo-controlled study. J dermatol 2004; 31(8):62731.

26. Brady S, Wilson JD. Closing the feedback loop: an audit of the use of imiquimod for the treatment of genital warts. J Eur Acad Dermatol Venereol 2004; 18(3):314-7.

27. Cusini M, Salmaso F, Zerboni R, Carminati G, Vernaci C, Franchi $\mathrm{C}$ et al. 5\% Imiquimod cream for external anogenital warts in HIV-infected patients under HAART therapy. Int J STD AIDS 2004; 15(1):17-20.

28. Nadal SR, Manzione CR. Doente portador de condiloma acuminado perianal recidivante: o que fazer? Rev Assoc Med Bras 2001; 47(4):278-279.

29. Loo WJ, Holt PJ. Bowenoid papulosis successfully treated with imiquimod. J Eur Acad Dermatol Venereol 2003; 17(3):3635 .

\section{Endereço para correspondência:} SIDNEY ROBERTO NADAL

Rua Mateus Grou, 130

05415-040 - São Paulo / SP

Fone/Fax: (11) 30824942

E-mail: srnadal@terra.com.br 